\title{
Insight into functionalized DMN-co-GMA copolymers
}

\author{
Part I. Modification with diethylenetriamine
}

\author{
Małgorzata Maciejewska ${ }^{1}$ (D) Magdalena Rogulska $^{1}$
}

Received: 7 November 2018 / Accepted: 27 April 2019/Published online: 13 May 2019

(c) The Author(s) 2019

\begin{abstract}
Suspension-emulsion polymerization was applied to obtain porous microspheres of 2,3-epoxypropyl methacrylate (GMA) cross-linked with 1,4-di(methacryloyloxymethyl)-naphthalene (DMN). In the next step, the porous methacrylate network was modified by subsequent reaction with diethylenetriamine. The newly obtained functionalized material was characterized in detail. After the process of modification, a drop in the value of specific surface area of the functionalized copolymers was observed. Their thermal behavior was monitored by TG/DSC/FTIR methods in inert and oxidative conditions. The reaction with diethylenetriamine resulted in the decrease in thermal resistance of the obtained porous microspheres. Initial decomposition temperatures (IDTs) established in helium atmosphere fell down from $258-269{ }^{\circ} \mathrm{C}$ for parent copolymers to $206-220{ }^{\circ} \mathrm{C}$ for modified ones. The same situation was observed in synthetic air atmosphere. IDTs decreased from 249-269 to $207-230^{\circ} \mathrm{C}$. Moreover, the thermal decomposition of the functionalized microspheres exhibited multistage decomposition patterns different from parent GMA-DMN copolymers.
\end{abstract}

Keywords Porous polymers · Microstructure $\cdot$ Thermal properties $\cdot$ TG/DSC/FTIR

\section{Introduction}

One of the most interesting types of materials is polymers with a permanent porosity. This feature, combined with chemical and thermal resistance, makes it possible to use them in miscellaneous adsorption techniques. Porous polymers are used as packing for HPLC [1-4], GC [5] and SPE [6-8] columns and also as specific adsorbents [9-14], ion exchangers $[15,16]$, cell culture media, blood flow indicators, peptide synthesis media, adsorbents in extracorporeal therapy, carriers of biologically active substances $[17,18]$, catalysts [19-21], etc. In comparison with commonly used adsorbents based on silica gel, they are characterized by the stability in the whole $\mathrm{pH}$ range, simplicity of introduction of functional groups, ability to create places for specific sorption. They combine in their structure the

Małgorzata Maciejewska

mmacieje@umcs.pl

1 Faculty of Chemistry, Maria Curie-Skłodowska University, pl. M. Curie-Skłodowskiej 3, 20-031 Lublin, Poland most desirable features of polymers and porous materials. Especially interesting are adsorbents of a polar nature that have affinity for a wide range of organic compounds. However, the direct synthesis of polar adsorbents is very often limited due to differences in the reactivity ratios of functional monomers and commonly used cross-linking agents. To overcome this limitation, monomers with oxirane ring are applied in the synthesis. Due to high steric stresses, the three-membered oxirane ring belongs to very reactive systems, and consequently, multi-directional chemical modification of synthesized polymers becomes possible. The newly created materials have different physical and chemical properties.

The main aim of this paper was the chemical modification of porous microspheres of 2,3-epoxypropyl methacrylate (GMA) cross-linked with 1,4-di(methacryloyloxymethyl) naphthalene (DMN) by diethylenetriamine (A) and next the determination of their thermal properties by means of DSC and TG coupled with FTIR. Additionally, the textural characterization was carried out on the basis of the low-temperature nitrogen adsorptiondesorption. 


\section{Experimental}

\section{Chemicals}

GMA from Sigma-Aldrich was washed with 5\% aqueous sodium hydroxide in order to remove inhibitors. Bis(2ethylhexyl) sulfosuccinate sodium salt (DAC, BP) and $\alpha, \alpha^{\prime}$ azoisobutyronitrile (AIBN) from Fluka AG (Buchs, Switzerland) and diethylenetriamine from Sigma-Aldrich were used without purification. Toluene and methyl alcohol (reagent grade) were purchased from POCh (Gliwice, Poland), whereas DMN was obtained in our laboratory according to the procedure described elsewhere [22-24].

\section{Preparation and modification of the GMA-co- DMN microspheres}

Copolymerization was performed in an aqueous suspension medium. $195 \mathrm{~mL}$ of distilled water and $2.2 \mathrm{~g}$ of DAC, BP were stirred for $2 \mathrm{~h}$ at $80{ }^{\circ} \mathrm{C}$ in order to dissolve the surfactant. Then the solution containing $15 \mathrm{~g}$ of monomers (GMA and DMN) and $0.2 \mathrm{~g}$ of AIBN dissolved in $22.5 \mathrm{~mL}$ of toluene was prepared and added while stirring to the aqueous medium. Molar ratios of GMA to DMN were changed from 1:1 to 5:1. Copolymerization was performed for $20 \mathrm{~h}$ at $80{ }^{\circ} \mathrm{C}$. Porous beads formed in this process were filtered off, and an extensive cleaning procedure was applied in order to remove the diluent unreacted monomers and physically adsorbed stabilizer. The cleaning process was as follows: The microspheres were separated from the aqueous phase by filtration of the polymerization mixture by $5 \mu \mathrm{m}$ filter papers. The microspheres were first washed with water, and the polymeric aggregates were removed by sieving. The microspheres were dispersed in water, and the dispersion was sonificated for $0.5 \mathrm{~h}$ in an ultrasonic bath. Next, the water phase was removed and the microspheres were resuspended in methyl alcohol. This dispersion was sonificated for $1 \mathrm{~h}$. Methyl alcohol was removed, and the microspheres were transferred into toluene and were kept there by stirring about $0.5 \mathrm{~h}$. Then, the toluene was removed, and microspheres were stirred with methyl alcohol for about $0.5 \mathrm{~h}$. Methyl alcohol was removed, and the microspheres were washed with distilled water, filtered and dried in vacuum oven at $65{ }^{\circ} \mathrm{C}$ for $48 \mathrm{~h}$.

The epoxy groups present in the copolymers were modified by the reaction with diethylenetriamine (Fig. 1). The procedure was as follows: In a $250-\mathrm{cm}^{3}$ round-bottomed two-necked flask equipped with a mechanical stirrer and a thermometer, $10 \mathrm{~g}$ of selected beads was placed together with diethylenetriamine and $200 \mathrm{~mL}$ of toluene, and the whole was heated in a water bath at $80{ }^{\circ} \mathrm{C}$ for $24 \mathrm{~h}$. The modified beads obtained were washed with distilled water, collected by filtration, dried and extracted in a Soxhlet apparatus with boiling toluene for $5 \mathrm{~h}$. The product was then dried under reduced pressure at $30{ }^{\circ} \mathrm{C}$.

\section{Methods of analysis}

Textural characterization of the copolymers was carried out by the low-temperature nitrogen adsorption-desorption method. Nitrogen adsorption-desorption isotherms were obtained at the liquid nitrogen temperature using a volumetric adsorption analyzer ASAP 2405 (Micromeritics Inc., USA). The measurements of the porous structure of the copolymers were taken by outgassing of the samples at $140{ }^{\circ} \mathrm{C}$ for $2 \mathrm{~h}$. The specific surface area of the investigated samples was calculated by the Brunauer-Emmet-Teller (BET) method for the adsorption data in the range of a relative pressure $p / p o 0.05$ to 0.25 . The total pore volume was estimated from a single point adsorption at a relative pressure of 0.985 . The pore size was obtained from the desorption branch of the isotherm using the Barrett-Joyner-Halenda $(\mathrm{BJH})$ procedure.

The microspheres were imaged using a LEO $1430 \mathrm{VP}$ numerical scanning electron microscope (SEM) from Carl Zeiss (Germany) with a countershaft and an energy-dispersive X-ray detector.

Attenuated total reflectance Fourier transform infrared (ATR-FTIR) spectra were obtained with a Bruker Tensor 27 FTIR spectrometer (Germany). The FTIR spectra were recorded in the spectral range of $600-4000 \mathrm{~cm}^{-1}$ with 16 scans per spectrum with a resolution of $4 \mathrm{~cm}^{-1}$.

The thermal properties of the synthesized copolymers were evaluated on the basis of measurements performed using the STA449, F1 Jupiter analyzer from Netzsch (Günzbung, Germany). The procedure was as follows: About $10 \mathrm{mg}$ of the sample was placed in the pan and heated in helium or in synthetic air atmosphere at a rate of $10 \mathrm{~K} \mathrm{~min}^{-1}$ up to $1000{ }^{\circ} \mathrm{C}$. The initial decomposition temperature (IDT), the temperatures of $20 \%\left(T_{20 \%}\right)$ and $50 \%\left(T_{50 \%}\right)$ mass losses and the final decomposition temperature (FDT) were determined.

The gaseous decomposition products formed under the degradation of functionalized copolymers were analyzed by means of a Bruker Tensor 27 FTIR spectrometer (Germany) coupled online to a STA instrument by a Teflon transfer line. Each FTIR spectrum was gathered every $10{ }^{\circ} \mathrm{C}$ in the spectral range from 600 to $4000 \mathrm{~cm}^{-1}$ with a resolution of $4 \mathrm{~cm}^{-1}$.

Differential scanning calorimetric (DSC) measurements were taken with the Netzsch DSC 204 calorimeter (Germany) operating in the dynamic mode. The dynamic scans were performed at the heating rate of $10 \mathrm{~K} \mathrm{~min}^{-1}$ from room temperature to the maximum of $500{ }^{\circ} \mathrm{C}$ under argon (30 $\mathrm{mL} \mathrm{min}^{-1}$ ) atmosphere. The mass of the sample was 

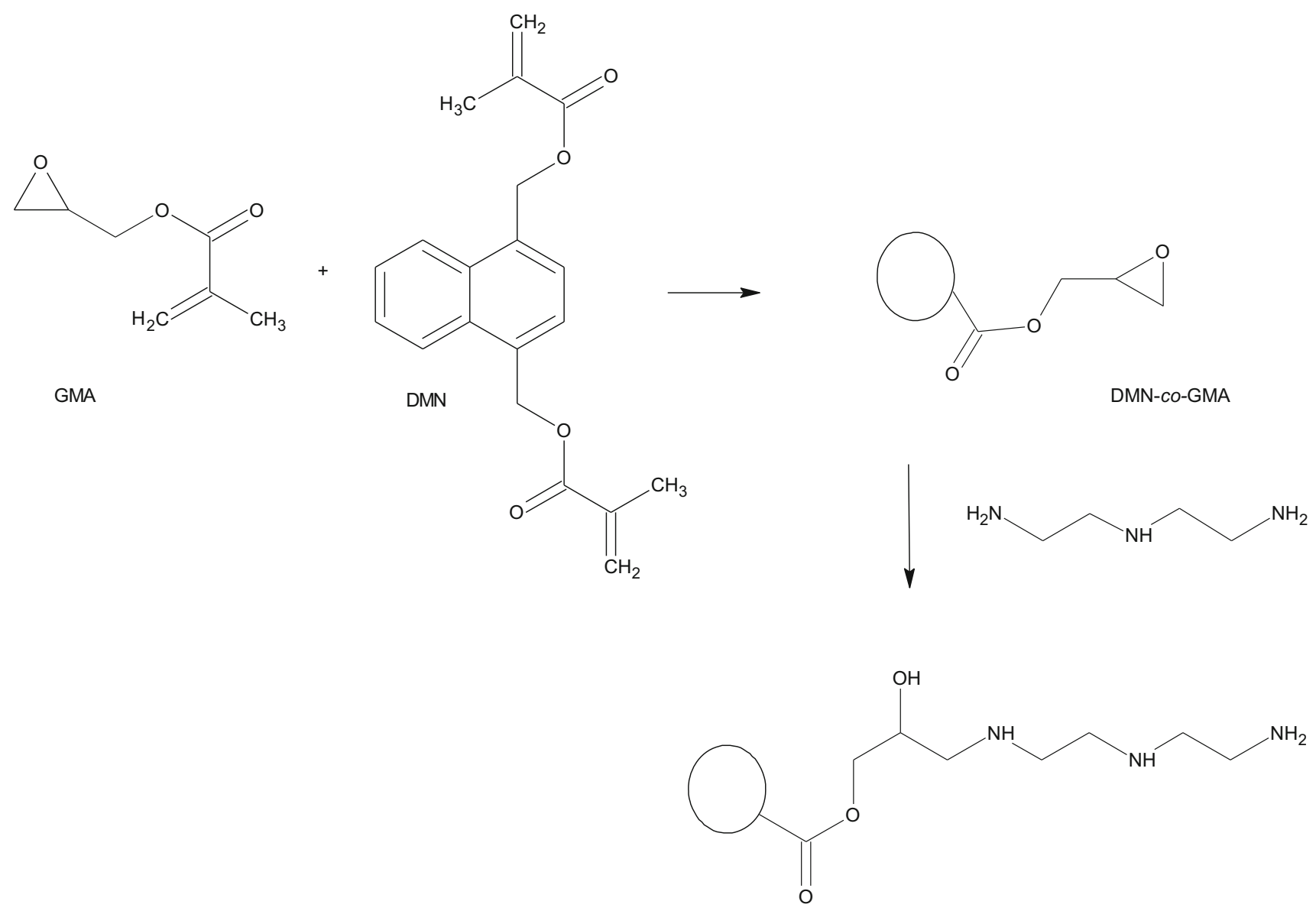

DMN-CO-GMA+A

Fig. 1 Scheme of the synthesis reaction of DMN-co-GMA copolymer and its modification with diethylenetriamine

about $10 \mathrm{mg}$. As a reference, an empty aluminum crucible was used.

\section{Results and discussion}

For the preparation of porous DMN-co-GMA copolymermodified suspension (called suspension-emulsion), polymerization was used. In this technique, the stabilizer of high molecular weights typical for suspension polymerization was replaced by an anionic surfactant-DAC, BP. The surfactant exceeded the critical micelle concentration (cmc), and the process of polymerization proceeded in the created micelles. As a result, regular microspheres were formed. In order to increase the amount of reactive epoxy group, the molar ratio of the functional monomer (GMA) to the cross-linker (DMN) was increased from 1:1 to 5:1.

In the next step of the investigation, a reaction with diethylenetriamine was conducted. During the reaction, epoxy groups present in the copolymer matrix were opened, and porous microspheres with pendant amine functionalities were obtained. Their diameters were in the range $20-50 \mu \mathrm{m}$ (Fig. 2). The symbols of newly created copolymers, based on the molar ratio of monomers in the polymerization mixture, are listed in Table 1 . The proper course of the reaction was also confirmed by the elemental analysis and ATR-FTIR spectroscopy. In the structure of the modified copolymers, considerable amount of nitrogen was found (Table 2). The content of nitrogen increases along with the enlargement of epoxy group that is capable of reacting with diethylenetriamine. Comparing the ATRFTIR spectra of modified and parent copolymers, disappearance of absorbance peaks originating from epoxide ring $\left(992,906\right.$ and $841 \mathrm{~cm}^{-1}$ ) is observed, whereas new peaks coming from amine group $\left(3352 \mathrm{~cm}^{-1}-\mathrm{N}-\mathrm{H}\right.$ stretching vibrations and $1571 \mathrm{~cm}^{-1}-\mathrm{N}-\mathrm{H}$ bending vibrations) and hydroxyl group $\left(3583 \mathrm{~cm}^{-1}-\mathrm{O}-\mathrm{H}\right.$ stretching vibrations and $1060 \mathrm{~cm}^{-1}-\mathrm{C}-\mathrm{OH}$ bending vibrations) are observed (Fig. 3).

As the investigated microspheres can be applied as adsorbents, it was also of interest to investigate their internal structure. Generally, the more the GMA monomer 


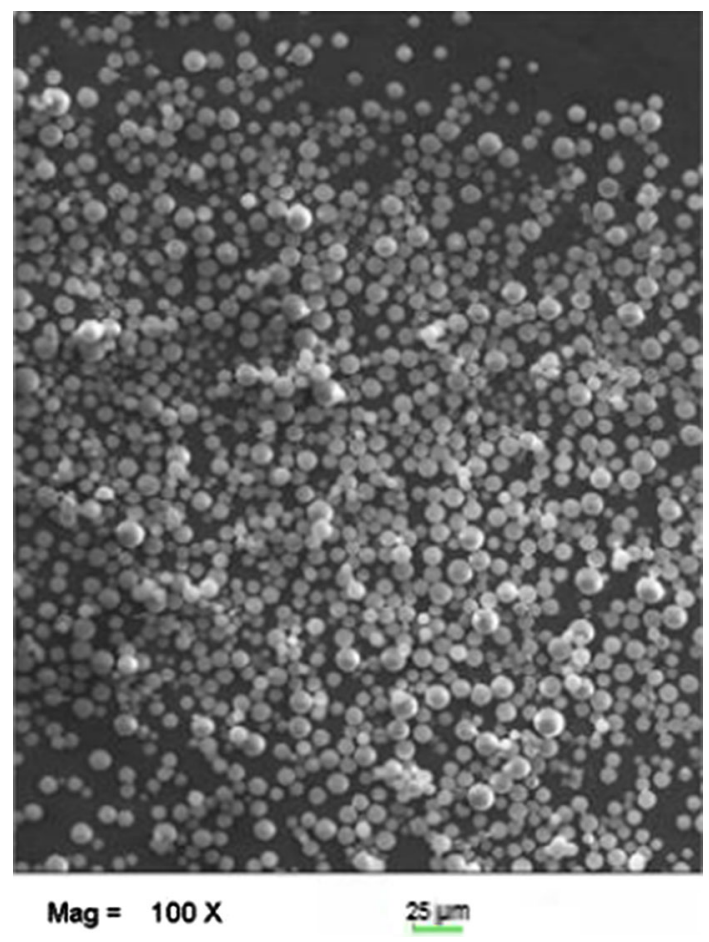

Fig. 2 SEM micrograph of functionalized DMN-co-GMA4+A copolymer

Table 1 Molar ratio of monomers used in the copolymerization

\begin{tabular}{lll}
\hline Copolymer & \multicolumn{2}{l}{ Molar ratio of monomers } \\
\cline { 2 - 3 } & DMN & GMA \\
\hline DMN-co-GMA1+A & 1 & 1 \\
DMN-co-GMA2+A & 1 & 2 \\
DMN-co-GMA3+A & 1 & 3 \\
DMN-co-GMA4+A & 1 & 4 \\
DMN-co-GMA5+A & 1 & 5 \\
\hline
\end{tabular}

in the polymerization mixture, the lower the values of specific surface area [25]. Additionally, the reaction with diethylenetriamine leads to noticeable decrease in the basic parameters of the internal structure of the modified copolymers. The value of specific surface area decreases from $106 \mathrm{~m}^{2} \mathrm{~g}^{-1}$ for DMN-co-GMA1 copolymer to $77 \mathrm{~m}^{2} \mathrm{~g}^{-1}$ for DMN-co-GMA1+A. Simultaneously the pore volume declines from 0.380 to $0.265 \mathrm{~cm}^{3} \mathrm{~g}^{-1}$ (Table 3). In the case of the least cross-linked DMN-coGMA5 copolymer, diminution of $S_{\mathrm{BET}}$ from 42 to $31 \mathrm{~m}^{2} \mathrm{~g}^{-1}$ and pore volume from 0.305 to 0.213 are observed. Bimodal pore size distribution for DMN-coGMA1 maintains its character after modification, but the maxima determined for parent copolymer (4 and $25 \mathrm{~nm}$ ) are shifted toward smaller values for functionalized one ( 3.5 and $12 \mathrm{~nm}$ ). For the remaining copolymers, only one maximum of pore size distribution is noticed. Nevertheless, the tendency of the decrease in its value after the process of modification is continued (Fig. 4).

The process of modification of the DMN-co-GMA copolymers with diethylenetriamine also contributes to the changes in the thermal properties of the newly obtained materials in both atmospheres. Figure 5 presents the thermogravimetric (TG) curves of DMN-co-GMA1 and DMNco-GMA1+A copolymers as an example.

As can be seen, the modification process leads to significant drop in the thermal resistance of the copolymers with amine functionalities. The IDT for the measurements conducted in helium decreases from $269^{\circ} \mathrm{C}$ determined for the DMN-co-GMA1 copolymer [25] to $220{ }^{\circ} \mathrm{C}$ for DMNco-GMA $1+\mathrm{A}$ one. For the remaining copolymers of the investigated series, the drop is even more notable. The IDTs fall from $258-268{ }^{\circ} \mathrm{C}$ for the parent copolymers to 206-208 ${ }^{\circ} \mathrm{C}$ for modified ones (Table 4). This phenomenon can be caused by the increased amine content in the functionalized copolymers. The same tendency is observed in the case of analysis conducted in the atmosphere of synthetic air. IDT for the DMN-co-GMA1+A copolymer is equal to $230{ }^{\circ} \mathrm{C}$ comparing to $269{ }^{\circ} \mathrm{C}$ for unmodified DMN-co-GMA1 one. For the rest of the investigated copolymers (Table 5), the decrease is at the similar level. TG and differential TG (DTG) curves of the all investigated DMN-co-GMA+A copolymers obtained in helium are shown in Fig. 6. The decomposition process in helium proceeds in three (with the exception of DMN-coGMA5+A) partially superimposed stages.
Table 2 Elemental analysis of the copolymers under study

\begin{tabular}{|c|c|c|c|c|c|c|}
\hline \multirow[t]{3}{*}{ Copolymer } & \multicolumn{6}{|c|}{ Element mass fraction/mass $\%$} \\
\hline & \multicolumn{2}{|l|}{$\mathrm{C}$} & \multicolumn{2}{|l|}{$\mathrm{H}$} & \multicolumn{2}{|l|}{$\mathrm{N}$} \\
\hline & Calculated & Determined & Calculated & Determined & Calculated & Determined \\
\hline $\mathrm{DMN}-$ co-GMA1+A & 64.90 & 63.97 & 8.26 & 7.17 & 7.32 & 4.17 \\
\hline DMN-co-GMA2+A & 61.90 & 60.20 & 8.16 & 7.50 & 10.31 & 5.13 \\
\hline $\mathrm{DMN}-c o-\mathrm{GMA} 3+\mathrm{A}$ & 60.04 & 58.93 & 8.46 & 7.66 & 11.89 & 5.56 \\
\hline DMN-co-GMA4+A & 58.87 & 57.79 & 8.65 & 7.79 & 12.87 & 6.52 \\
\hline DMN-co-GMA5+A & 58.08 & 55.88 & 8.77 & 7.86 & 13.55 & 6.98 \\
\hline
\end{tabular}


Fig. 3 ATR-FTIR spectra of parent (DMN-co-GMA5) and modified with diethylenetriamine (DMN-coGMA5+A) copolymers

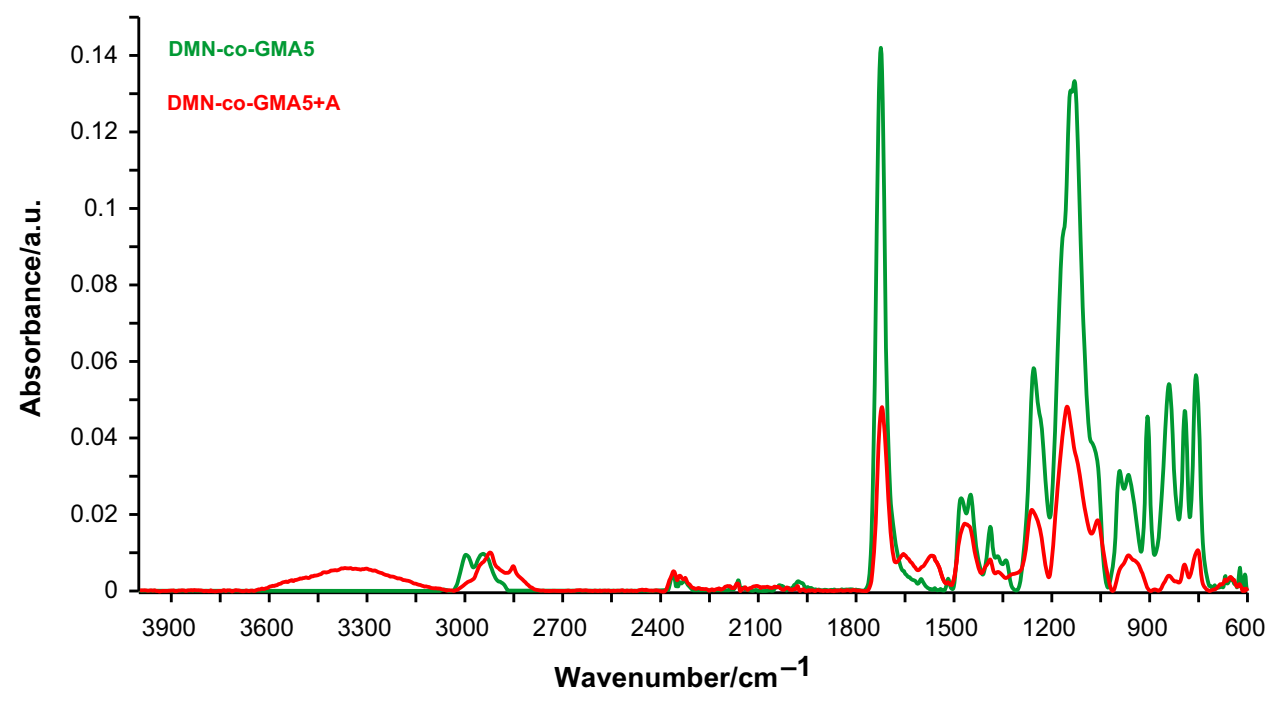

Table 3 Basic parameters of porous structure of the copolymers

\begin{tabular}{llll}
\hline Copolymer & Specific surface area $\left(S_{\mathrm{BET}}\right) / \mathrm{m}^{2} \mathrm{~g}^{-1}$ & Pore volume $(V) / \mathrm{cm}^{3} \mathrm{~g}^{-1}$ & Pore diameter $\left(D_{\mathrm{BJH}}\right) / \mathrm{nm}$ \\
\hline DMN-co-GMA1+A & 77 & 0.265 & $3.5 / 12$ \\
DMN-co-GMA2+A & 66 & 0.410 & 31 \\
DMN-co-GMA3+A & 54 & 0.406 & 44 \\
DMN-co-GMA4+A & 44 & 0.303 & 46 \\
DMN-co-GMA5+A & 31 & 0.213 & 43 \\
\hline
\end{tabular}

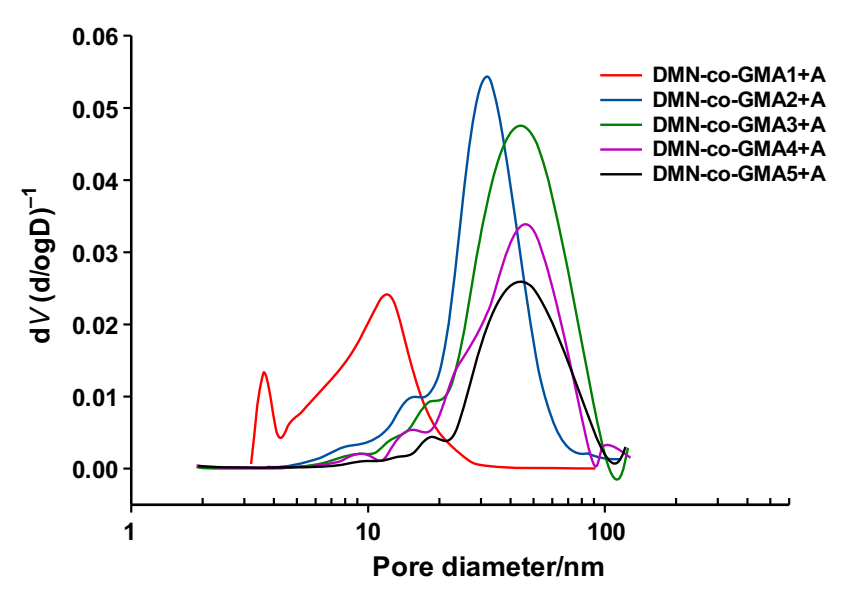

Fig. 4 Pore size distribution of modified DMN-co-GMA copolymers

The analysis of the chemical structure of DMN-coGMA+A copolymers suggests that both $\alpha$-hydrogen bond scission (formation and emission of aldehydes) and $\beta$-hydrogen bond scission (formation and emission of carboxylic and vinyl compounds) are possible. However, in the case of highly cross-linked copolymers the random chain scission is also probable. The first decomposition stage with the maxima in the range of $260-280{ }^{\circ} \mathrm{C}$ can be associated with the degradation process of epoxy rings that are still present in the polymer network. At the second one with the maxima in the range of $346-380{ }^{\circ} \mathrm{C}$, mainly chain scission of ester bonds may occur. The highest maximum temperature of this step is observed for the most crosslinked copolymer (DMN-co-GMA1+A) that contains the largest amount of ester bonds. At the third stage (403-412 ${ }^{\circ} \mathrm{C}$ ), thermal degradation of cross-linked parts of the copolymers may take place. What is interesting, in the case of the copolymer with the highest content of amine functionalities (DMN-co-GMA5+A) the second and third stages are coupled in one with the maximum at $403{ }^{\circ} \mathrm{C}$.

Figure 7 displays TG and DTG curves for all DMN-coGMA+A copolymers obtained in synthetic air. Comparing with helium atmosphere, the curves are more diversified. The decomposition process proceeds in four stages, and the difference between the individual copolymers is more visible. The IDTs for all copolymers under study are higher than temperatures determined in helium. The same situation is observed for the FDTs. Additional stage in the decomposition can be attributed to oxidative degradation of ester bonds.

The proposed course of the decomposition process is reflected in the analysis of the volatile decomposition 
Fig. 5 TG curves of parent (DMN-co-GMA1) and modified with diethylenetriamine (DMNco-GMA1+A) copolymers obtained in helium and synthetic air atmospheres
Table 4 Thermal stability of the copolymers under study determined in helium

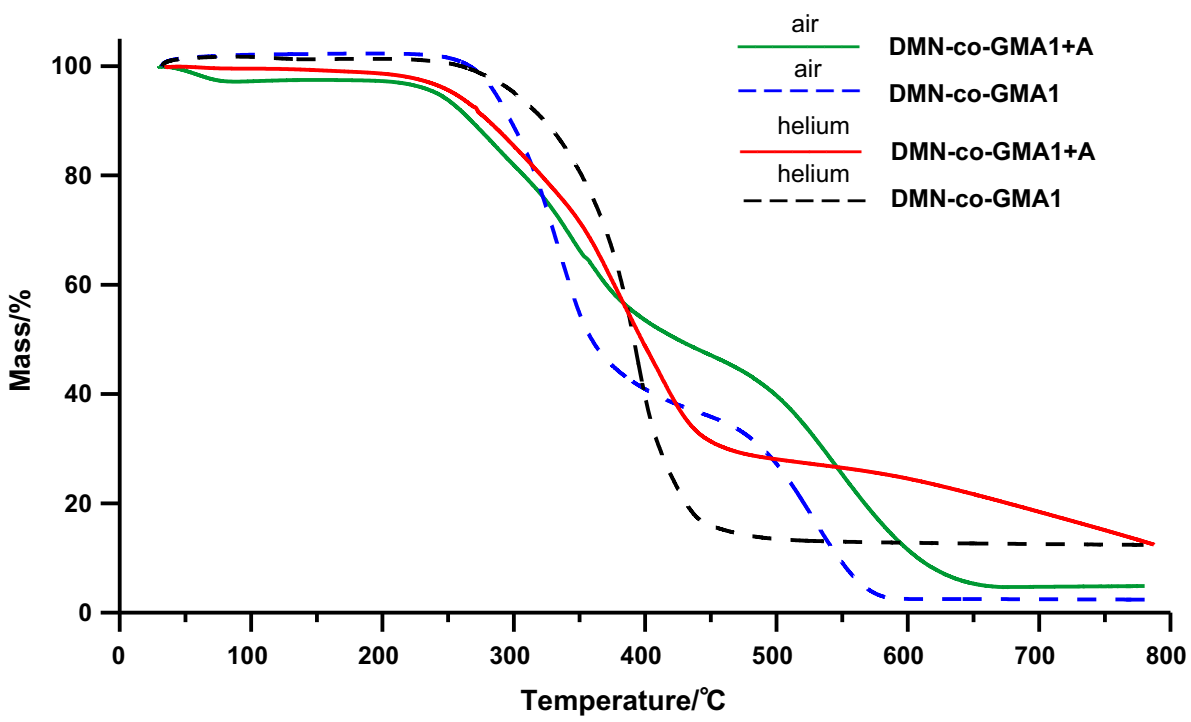

\begin{tabular}{llllllll}
\hline Copolymer & $\mathrm{IDT} /{ }^{\circ} \mathrm{C}$ & $T_{20 \%} /{ }^{\circ} \mathrm{C}$ & $T_{50 \%} /{ }^{\circ} \mathrm{C}$ & $\mathrm{FDT} /{ }^{\circ} \mathrm{C}$ & $T_{1 \max } /{ }^{\circ} \mathrm{C}$ & $T_{2 \max } /{ }^{\circ} \mathrm{C}$ & $T_{3 \max } /{ }^{\circ} \mathrm{C}$ \\
\hline DMN-co-GMA1+A & 220 & 322 & 398 & 516 & 280 & 380 & 407 \\
DMN-co-GMA2+A & 208 & 311 & 396 & 498 & 265 & 346 & 412 \\
DMN-co-GMA3+A & 207 & 303 & 393 & 498 & 260 & 356 & 405 \\
DMN-co-GMA4+A & 206 & 300 & 392 & 496 & 260 & 360 & 412 \\
DMN-co-GMA5+A & 206 & 298 & 387 & 491 & 260 & - & 403 \\
\hline
\end{tabular}

Table 5 Thermal stability of the copolymers under study determined in synthetic air

\begin{tabular}{|c|c|c|c|c|c|c|c|c|}
\hline Copolymer & $\mathrm{IDT} /{ }^{\circ} \mathrm{C}$ & $T_{20 \%} /{ }^{\circ} \mathrm{C}$ & $T_{50 \%} /{ }^{\circ} \mathrm{C}$ & $\mathrm{FDT} /{ }^{\circ} \mathrm{C}$ & $T_{1 \max } /{ }^{\circ} \mathrm{C}$ & $T_{2 \max } /{ }^{\circ} \mathrm{C}$ & $T_{3 \max } /{ }^{\circ} \mathrm{C}$ & $T_{4 \max } /{ }^{\circ} \mathrm{C}$ \\
\hline $\mathrm{DMN}-$ co-GMA1+A & 230 & 319 & 450 & 664 & 270 & 285 & 344 & 540 \\
\hline $\mathrm{DMN}-c o-\mathrm{GMA} 2+\mathrm{A}$ & 225 & 300 & 413 & 654 & 257 & 308 & 362 & 524 \\
\hline DMN-co-GMA3+A & 216 & 285 & 360 & 651 & 255 & 312 & 343 & 509 \\
\hline DMN-co-GMA4+A & 207 & 281 & 364 & 660 & 259 & 323 & 343 & 519 \\
\hline DMN-co-GMA5+A & 211 & 282 & 365 & 660 & 256 & 324 & 344 & 518 \\
\hline
\end{tabular}

products. The analysis was conducted for the selected copolymer, i.e., DMN-co-GMA4+A. Figure 8 displays 3D diagrams of FTIR spectra of gases evolved during thermal decomposition of this copolymer in helium and synthetic air atmospheres. It is clearly visible that at temperatures ranging from 40 to $260{ }^{\circ} \mathrm{C}$ only the moisture vaporization from the studied materials is observed. As the temperature increased, the formation of additional gaseous products was observed. The maxima of gases emissions are in accordance with the maxima determined from DTG curves. For the detailed discussion of the obtained results, the maxima were extracted and are shown in Figs. 9 and 10.

At the emission spectrum collected in helium (see Fig. 9) at the first maximum of decomposition (temperature $260{ }^{\circ} \mathrm{C}$ ), bands connected with the evolution of unsaturated aliphatic compounds (at $\sim 3070$ to $2850 \mathrm{~cm}^{-1}$, attributed to the $\mathrm{C}-\mathrm{H}$ stretching vibrations of methylene, methyl and vinyl groups and at 965 and $931 \mathrm{~cm}^{-1}$, related to the $\mathrm{C}-\mathrm{H}$ out-of-plane deformation vibrations of vinyl group) are visible. They can suggest the $\beta$-elimination decomposition of epoxide and ester functionalities. What is more, bands connected with carbonyl compounds including esters (bands at $\sim 1750 \mathrm{~cm}^{-1}$, characteristic of the $\mathrm{C}=\mathrm{O}$ stretching vibrations and at $1158 \mathrm{~cm}^{-1}$, connected with the C-O stretching vibrations of the ester groups), carbon dioxide (bands at 2359-2310 $\mathrm{cm}^{-1}$, attributed to the asymmetric stretching vibrations and at $669 \mathrm{~cm}^{-1}$, associated with the degenerate bending vibrations) and evolution of water (bands at $\sim 4000$ to $3500 \mathrm{~cm}^{-1}$, connected 


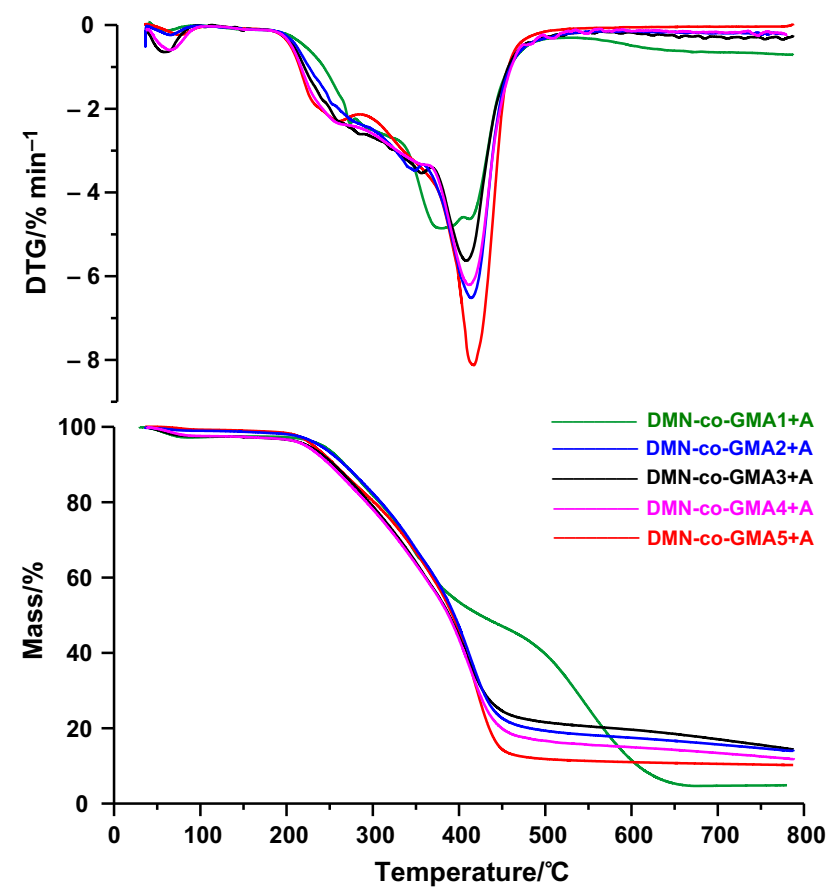

Fig. 6 TG and DTG curves for all DMN-co-GMA+A copolymers obtained in helium atmosphere

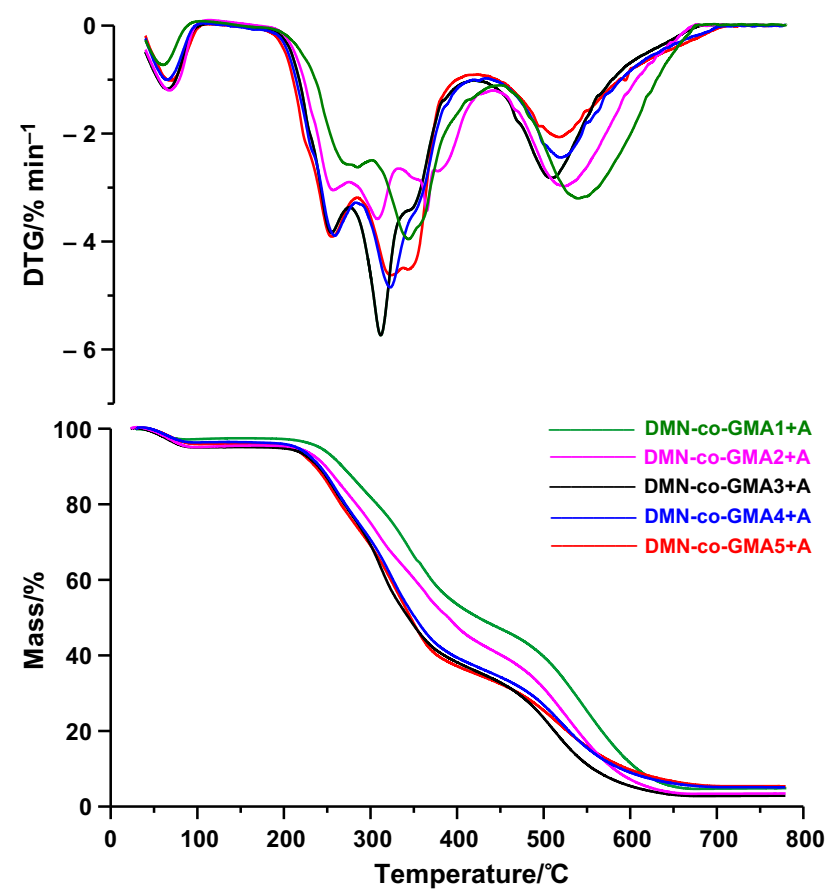

Fig. 7 TG and DTG curves for all DMN-co-GMA+A copolymers obtained in synthetic air atmosphere

with the stretching vibrations and at $\sim 1800$ to $1300 \mathrm{~cm}^{-1}$ — with the bending vibrations) can be found.

In the second stage (the maximum at $356{ }^{\circ} \mathrm{C}$ ), the same bands as in the previous one are observed, but their intensity is much higher. Also bands pointing to the formation of carbon monoxide (bands at 2181 and $2114 \mathrm{~cm}^{-1}$, related to the stretching vibrations) appear. Creating unsaturated aliphatic compounds is confirmed by the presence of the bands at $1460-1300 \mathrm{~cm}^{-1}$ that correspond to the deformation vibrations of methylene, methyl and vinyl groups. The absorption band at $3582 \mathrm{~cm}^{-1}$ (related to the $\mathrm{O}-\mathrm{H}$ stretching vibrations) indicates the emission of allyl alcohol or glycidol that can be the products of ester and epoxide decomposition [26]. Additionally, bands indicated different types of carbonyl compounds (at 1765 and $1732 \mathrm{~cm}^{-1}$ ) are present. Apart from esters, also the aldehydes can be emitted what is confirmed by the bands in the region of $\sim 2800$ to $2700 \mathrm{~cm}^{-1}$. This phenomenon indicates that $\alpha$-hydrogen bond scission degradation can occur in the case of DMN-co-GMA+A copolymers.

The next maximum of emission of gases formed during the copolymer decomposition was observed at $412{ }^{\circ} \mathrm{C}$. At this temperature, mainly the cross-linked parts of the sample decomposed, and in consequence, significant evolution of carbon dioxide (bands at $2359-2310 \mathrm{~cm}^{-1}$, attributed to the asymmetric stretching vibrations and at $669 \mathrm{~cm}^{-1}$, associated with the degenerate bending vibrations) and carbon monoxide (bands at 2181 and $2114 \mathrm{~cm}^{-1}$, related to the stretching vibrations) connected with decarboxylation processes was observed.

As the three stages are partially superimposed, the same products of the chain scission can appear in each of them.

In oxidative conditions, the obtained spectra (Figs. 8, 10) are less diversified compared to the spectra received in inert conditions. At first, second and third stages (the maxima at 259,323 and $343{ }^{\circ} \mathrm{C}$, respectively), bands confirming $\alpha$-hydrogen and $\beta$-hydrogen bond scission can be found, leading to the formation of unsaturated aliphatic compounds (bands at $936 \mathrm{~cm}^{-1}$, attributed to the $\mathrm{C}-\mathrm{H}$ deformation vibration of vinyl group, at $1650 \mathrm{~cm}^{-1}$, related to the $\mathrm{C}=\mathrm{C}$ stretching vibrations and at $3095 \mathrm{~cm}^{-1}$ - to the $\mathrm{C}-\mathrm{H}$ and $=\mathrm{CH}_{2}$ stretching vibrations), carbonyl compounds (bands at $1793-1740 \mathrm{~cm}^{-1}$, connected with the $\mathrm{C}=\mathrm{O}$ stretching vibrations), other aldehydes (bands at 2819 and $2748 \mathrm{~cm}^{-1}$, associated with the $\mathrm{C}-\mathrm{H}$ stretching vibrations) and carboxylic spices, including esters (bands at $1156-1134 \mathrm{~cm}^{-1}$, connected with the $\mathrm{C}-\mathrm{O}$ stretching vibrations). It can be also noticed that the greatest amount of those products, with the exception of carbon dioxide, was evolved during the first decomposition stage. Moreover, in the spectra the band characteristics of water and carbon monoxide are visible. At the last stage with the maximum at $521{ }^{\circ} \mathrm{C}$ only water, carbon monoxide and a large amount of carbon dioxide were formed.

Figure 11 presents the Gram-Schmidt plots obtained during the thermal decomposition for parent and modified copolymers in helium. On the curve originated from DMN- 


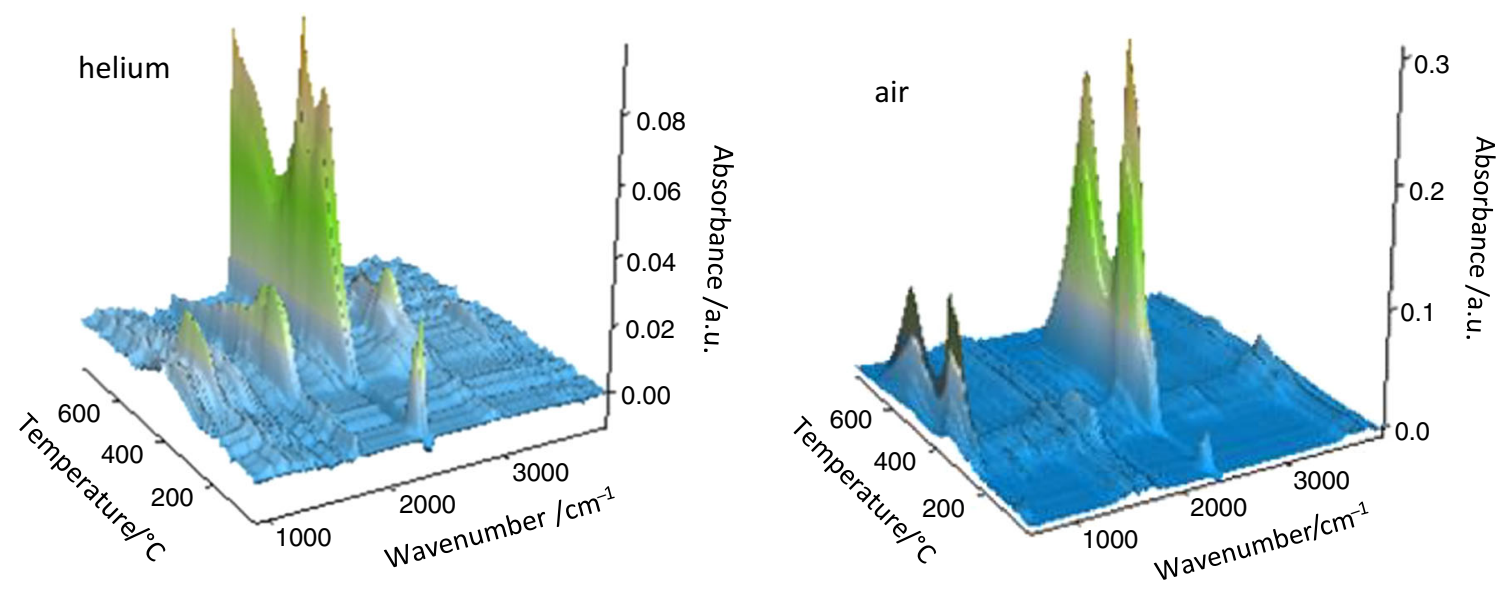

Fig. 8 3D diagrams of FTIR spectra of gases evolved during thermal decomposition of DMN-co-GMA4+A copolymer in helium and synthetic air atmospheres

Fig. 9 FTIR spectra of gases evolved from DMN-co-

GMA4+A copolymer at the maxima of decomposition in helium atmosphere

Fig. 10 FTIR spectra of gases evolved from DMN-coGMA4+A copolymer at the maxima of decomposition in synthetic air atmosphere
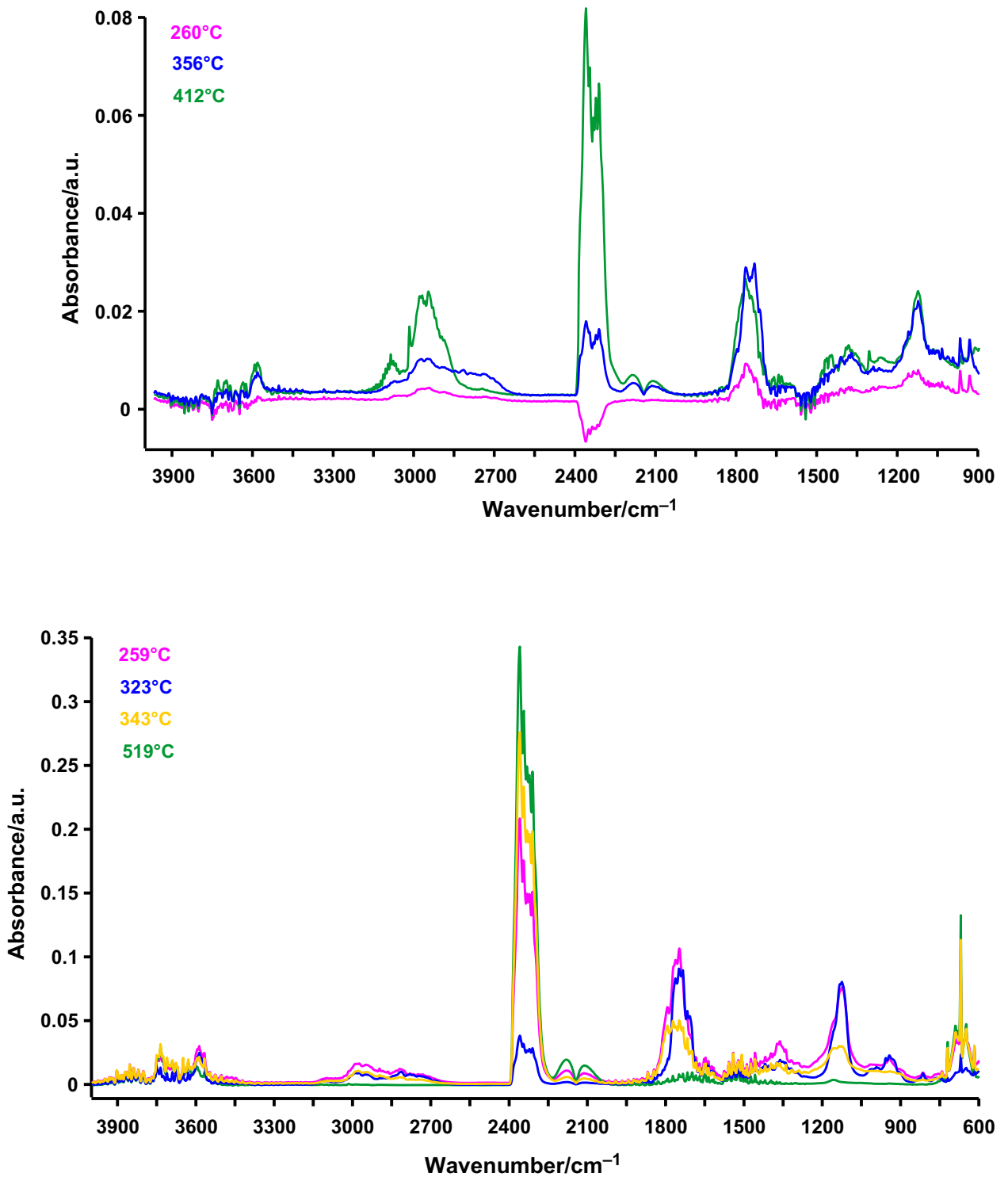


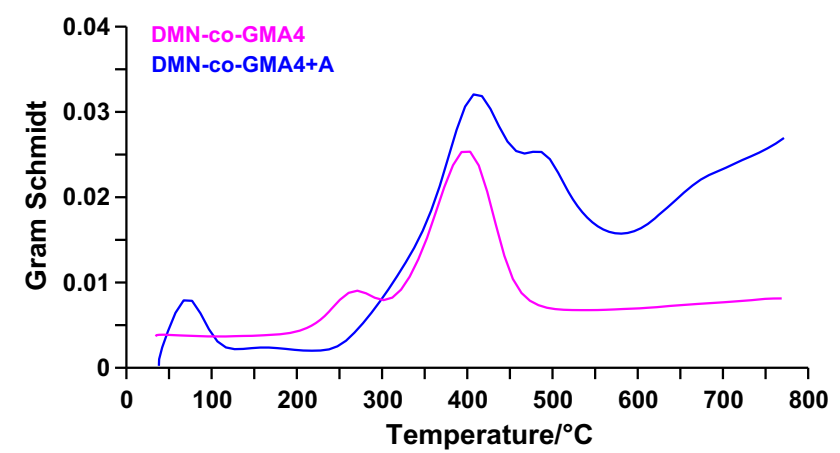

Fig. 11 Gram-Schmidt plots of parent (DMN-co-GMA4) and modified diethylenetriamine (DMN-co-GMA4+A) copolymers in helium atmosphere

co-GMA4+A copolymer, three main peaks are visible with reference to two exhibited on the DMN-co-GMA4 one. The first with the maximum at $75{ }^{\circ} \mathrm{C}$ is connected with the evolution of water adsorbed by more polar surface of DMN-GMA4+A copolymer, whereas the second with the maximum at $420{ }^{\circ} \mathrm{C}$ is associated with the decomposition of cross-linked polymeric matrix. In turn, the first peak on DMN-co-GMA4 curve refers to the decomposition of epoxy groups that are not present in the case of the modified copolymer. On the other hand, the modified copolymers possess amine functionalities that contribute to another additional peak with the maximum at $510{ }^{\circ} \mathrm{C}$.

Thermal behavior of the discussed copolymers was also investigated by means of the DSC method. Figure 12 shows DSC curves for all functionalized copolymers obtained in helium atmosphere. By looking at the curves, few thermal events can be seen, both exothermic and endothermic ones. The first endothermic peak on the DSC curve can be attributed to the process of moisture vaporization from the studied materials. Along with the increase in the amount of amine functionalities in the copolymer, the amount of adsorbed water significantly increases. The second endothermic peak with the maximum at 267-279 ${ }^{\circ} \mathrm{C}$ (depending on the copolymer) can be attributed to the degradation reaction of ester groups. The third endothermic peak with the maximum at $408-412{ }^{\circ} \mathrm{C}$ indicates the thermal degradation of the cross-linked parts of the copolymers. Also the exothermic reactions in the thermal treatment of DMN-co-GMA+A copolymers can be seen on the DSC curves. The first exothermic peak at $217-221^{\circ} \mathrm{C}$ could be caused by pendant double bonds. Highly cross-linked porous copolymers can contain considerable amount of unreacted double bonds that can undergo reaction of thermal polymerization. As a result, a peak indicating the exothermal reaction can be observed. The second exothermic peak following the first step of endothermic decomposition can be ascribed to a thermal

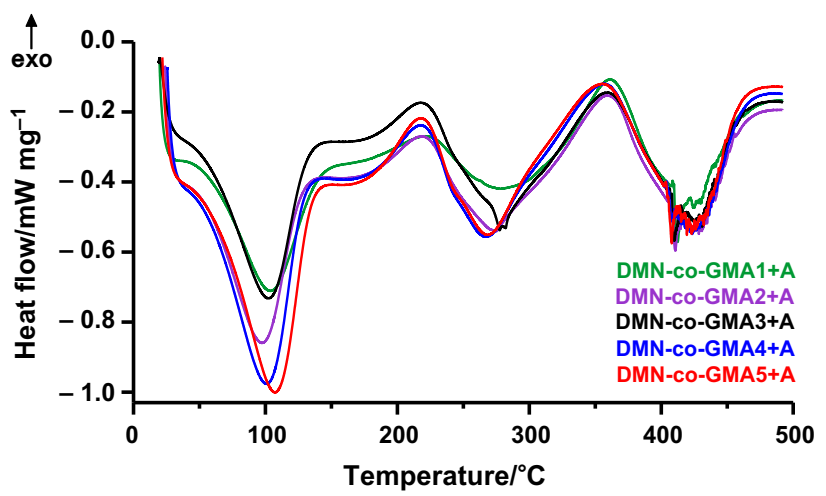

Fig. 12 DSC curves for all DMN-co-GMA+A copolymers obtained in helium atmosphere

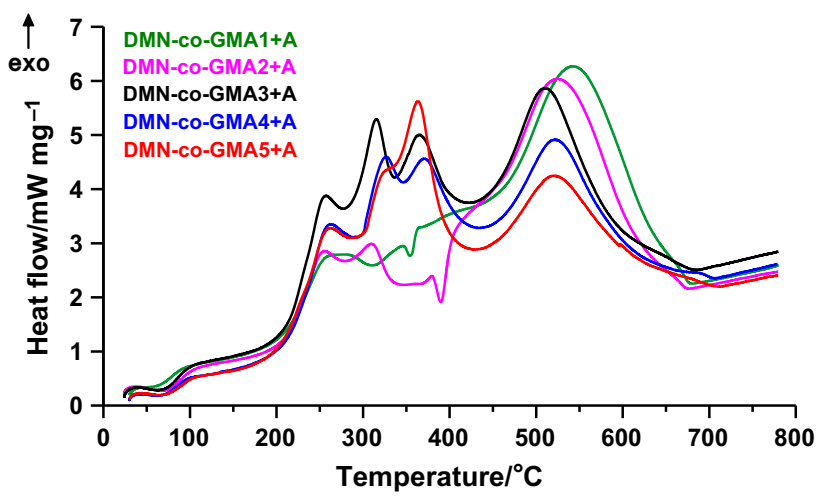

Fig. 13 DSC curves for all DMN-co-GMA+A copolymers obtained in synthetic air atmosphere

cross-linking reaction unreacted epoxide and incorporated amine groups with carboxylic acid groups, which are formed in the first step of thermal degradation by the random chain scission reactions.

In the synthetic air atmosphere, a series of exothermal events take place (Fig. 13). The first exothermic process (maximum at $256-262^{\circ} \mathrm{C}$ ) can be connected with the thermal polymerization of double bonds that are located close to each other. Another exothermic reaction (at $310-323{ }^{\circ} \mathrm{C}$ ) can be related to the double-bond oxidation, resulting in the formation of carbonyl groups. In the third step, the newly formed groups can undergo the exothermic cross-linking reaction. The last step is connected with the final oxidative decomposition.

\section{Conclusions}

In the present study, porous DMN-co-GMA copolymers were functionalized with diethylenetriamine. The newly obtained functionalized materials were characterized in detail. Their thermal behavior was monitored by TG/DSC/ FTIR methods in inert and oxidative atmospheres. The 
conducted studies lead to the conclusion that the process of functionalization strongly influenced the textural and thermal properties of the copolymers. The introduction of amine groups into methacrylate matrix induced the considerable drop in the value of the basic parameters of the internal structure. Also the thermal resistance of the newly obtained materials decreases. The IDTs established in helium atmosphere fell down from $258-269{ }^{\circ} \mathrm{C}$ for parent copolymers to $206-220{ }^{\circ} \mathrm{C}$ for modified ones. Analogical drop is observed in the case of synthetic air atmosphere. The examination of the DTG curves shows that the thermal decomposition of the functionalized copolymers conducted in helium proceeded in three stages, whereas in synthetic air it was in four ones. Moreover, on the basis of detailed analysis of the volatile decomposition products it can be stated that modified DMN-co-GMA+A copolymers can decompose according to the $\alpha$-hydrogen and $\beta$-hydrogen bond scission mechanisms. Random chain scission mechanism is also probable.

Acknowledgements The research was carried out with the equipment purchased thanks to the financial support of the European Regional Development Fund in the framework of the Operational Program Development of Eastern Poland 2007-2013 (Contract No. POPW.01.03.00-06-009/11-00, Equipping the laboratories of the Faculties of Biology and Biotechnology, Mathematics, Physics and Informatics, and Chemistry for studies of biologically active substances and environmental samples).

Open Access This article is distributed under the terms of the Creative Commons Attribution 4.0 International License (http://creative commons.org/licenses/by/4.0/), which permits unrestricted use, distribution, and reproduction in any medium, provided you give appropriate credit to the original author(s) and the source, provide a link to the Creative Commons license, and indicate if changes were made.

\section{References}

1. Grochowicz M, Szajnecki Ł, Gawdzik B. 4VP-TRIM composite polymer particles and their application as adsorbents. Adsorpt Sci Technol. 2015;33:609-16.

2. Maciejewska M, Osypiuk J, Gawdzik B. Preparation and characterization of chromatographic properties of ethylene glycol dimethacrylate-divinylbenzene polymeric microspheres. J Polym Sci Part A Polym Chem. 2005;43:3049-58.

3. Unsal E, Irmak T, Tuncel M. Monodisperse poly (styrene-codivinylbenzene) particles $(3.2 \mu \mathrm{m})$ with relatively small pore size as HPLC packing material. Chromatographia. 2004;60:553-60.

4. Benson JR, Woo DJ. Polymeric columns for liquid chromatography. J Chromatogr Sci. 1984;22:386-99.

5. Maciejewska M, Szajnecki $Ł$, Gawdzik B. Investigation of the surface area and polarity of porous copolymers of maleic anhydride and divinylbenzene. J Appl Polym Sci. 2012;125:300-7.

6. Puziy AM, Poddubnaya OI, Gawdzik B, Sobiesiak M, Reinish CA, Tsyba MM, Segeda TP, Danylenko MI. Nanostructured carbons for solid phase extraction. Appl Surf Sci. 2010;256:5216-20.
7. Sobiesiak M, Podkościelna B. Preparation and characterization of porous DVB copolymers and their applicability for adsorption (solid-phase extraction) of phenol compounds. Appl Surf Sci. 2010;257:1222-7.

8. Maciejewska M, Gawdzik J. Preparation and characterization of sorption properties of porous microspheres of 1-vinyl-2-pyrrolidone-divinylbenzene. J Liq Chromatogr Related Technol. 2008;31:950-61.

9. Bilgin Simsek E, Aytas B, Duranoglu D, Beker U, Trochimczuk AW. A comparative study of 2-chlorophenol, 2,4-dichlorophenol, and 2,4,6-trichlorophenol adsorption onto polymeric, commercial, and carbonaceous adsorbents. Desalin Water Treat. 2016;57:9940-56.

10. Xiao G, Wen R, You P, Wu D. Adsorption of phenol onto four hyper-cross-linked polymeric adsorbents: effect of hydrogen bonding receptor in micropores on adsorption capacity. Microporous Mesoporous Mater. 2017;239:40-4.

11. Kolarz BN, Jermakowicz-Bartkowiak D. Poly(4-vinylpyridine) resins towards perrhenate sorption and desorption. React Funct Polym. 2011;71:95-103.

12. Grochowicz M. Investigation of the thermal behavior of 4-vinylpyridine-trimethylolpropane trimethacrylate copolymeric microspheres. J Therm Anal Calorim. 2014;118:1603-11.

13. Tsyurupa MP, Davankov VA. Porous structure of hypercrosslinked polystyrene: state-of-the-art mini-review. React Funct Polym. 2006;66:768-79.

14. Xiao WT, Zhang ZJ. Fabrication of porous polymers. Prog Chem. 2009;21:1299-303.

15. Maciejewska M. Characterization of macroporous 1-vinyl-2pyrrolidone copolymers obtained by suspension polymerization. J Appl Polym Sci. 2012;124:568-75.

16. Legan M, Trochimczuk AW. Bis-imidazolium type ion-exchange resin with poly(HIPE) structure for the selective separation of anions from aqueous solutions. Sep Sci Technol. 2018;53:1163-77.

17. Kawaguchi H. Functional polymer microspheres. Prog Polym Sci. 2000;25:1171-210.

18. Jagur-Grodzinski J. Biomedical application of functional polymers. React Funct Polym. 1999;39:99-138.

19. Fan S, Luan Y, Wang J, Gao H, Zhang X, Wang G. Monodispersed poly(4-vinylpyridine) spheres supported $\mathrm{Fe}(\mathrm{III})$ material: an efficient and reusable catalyst for benzylic oxidation. J Mol Catal A Chem. 2015;404-405:186-92.

20. de Aguiar V, de Souza A, Galdino F, et al. Sulfonated poly(divinylbenzene) and poly(styrene-divinylbenzene) as catalysts for esterification of fatty acids. Renew Energ. 2017;114:725-32.

21. Kolarz BN, Jakubiak A. Catalytic activity of molecular imprinted vinylpyridine/acrylonitrile/divinylbenzene terpolymers with guanidyl ligands-Cu(II) inside the active centers. Polymers. 2008;53:848-53.

22. Gawdzik B, Czerwińska-Bil U. Porous copolymer-based cation exchanger for the off-line preconcentration of aromatic amines from water. Chromatography. 1991;32:167-70.

23. Matynia T, Gawdzik B. Influence of synthesis conditions of porous copolymers of 1,4-di(methacryloyloxymethyl) naphthalene with divinylbenzene on their structure. I. Influence of mol composition of monomers. Angew Makromol Chem. 1987; 147:123-32.

24. Gawdzik B, Matynia T. Influence of synthesis conditions of porous copolymers of 1,4-di(methacryloyloxymethyl) naphthalene with divinylbenzene on their structure. II. Influence of diluent composition. Angew Makromol Chem. 1987;125:33-5.

25. Maciejewska M. Synthesis and thermal properties of parent and modified DMN-co-GMA copolymers. J Therm Anal Calorim. 2018;133:969-80. 
26. Grochowicz M, Paczkowski P, Gawdzik B. Investigation of the thermal properties of glycidyl methacrylate-ethylene glycol dimethacrylate copolymeric microspheres modified by DielsAlder reaction. J Therm Anal Calorim. 2018;133:499-508.
Publisher's Note Springer Nature remains neutral with regard to jurisdictional claims in published maps and institutional affiliations. 